\title{
Duplications of BHLHA9 are associated with ectrodactyly and tibia hemimelia inherited in non-Mendelian fashion
}

Eva Klopocki, ${ }^{1,2}$ Silke Lohan, ${ }^{1,2}$ Sandra C Doelken, ${ }^{1}$ Sigmar Stricker,
${ }^{2}$
Charlotte W Ockeloen, ${ }^{3}$ Renata Soares Thiele de Aguiar, ${ }^{4}$ Karina Lezirovitz, ${ }^{4,5}$
Regina Celia Mingroni Netto, ${ }^{4}$ Aleksander Jamsheer, ${ }^{6,7}$ Hitesh Shah, ${ }^{8}$ Ingo Kurth,
Rolf Habenicht ${ }^{10}{ }^{9}$ Matthew Warman, ${ }^{11}$ Koenraad Devriendt, ${ }^{12}$ Ulrike Kordaß, ${ }^{13}$
Maja Hempel, ${ }^{14,15}$ Anna Rajab, ${ }^{16}$ Outi Mäkitie, ${ }^{17}$ Mohammed Naveed, ${ }^{18}$
Uppala Radhakrishna, ${ }^{19}$ Stylianos E Antonarakis, ${ }^{19,20}$ Denise Horn, ${ }^{1}$ Stefan Mundlos ${ }^{1,2}$

- Additional figures are published online only. To view these files please visit the journal online (http://jmg.bmj. com/content/49/2.toc).

For numbered affiliations see end of article.

\section{Correspondence to}

Dr Eva Klopocki, Institute for Medical Genetics and Human Genetics, Charité Universitätsmedizin Berlin, Augustenburger Platz 1, 13353 Berlin, Germany; eva.klopocki@charite.de

Received 8 August 2011 Revised 11 November 2011 Accepted 12 November 2011 Published Online First 6 December 2011

\begin{abstract}
Background Split-hand/foot malformation (SHFM) —also known as ectrodactyly - is a congenital disorder characterised by severe malformations of the distal limbs affecting the central rays of hands and/or feet. A distinct entity termed SHFLD presents with SHFM and long bone deficiency. Mouse models suggest that a defect of the central apical ectodermal ridge leads to the phenotype. Although six different loci/mutations (SHFM1-6) have been associated with SHFM, the underlying cause in a large number of cases is still unresolved.
\end{abstract}

Methods High resolution array comparative genomic hybridisation (CGH) was performed in patients with SHFLD to detect copy number changes. Candidate genes were further evaluated for expression and function during limb development by whole mount in situ hybridisation and morpholino knock-down experiments.

Results Array CGH showed microduplications on chromosome 17p13.3, a locus previously associated with SHFLD. Detailed analysis of 17 families revealed that this copy number variation serves as a susceptibility factor for a highly variable phenotype with reduced penetrance, particularly in females. Compared to other known causes for SHFLD 17p duplications appear to be the most frequent cause of SHFLD. A $\sim 11.8 \mathrm{~kb}$ minimal critical region was identified encompassing a single gene, BHLHA9, a putative basic loop helix transcription factor. Whole mount in situ hybridisation showed expression restricted to the limb bud mesenchyme underlying the apical ectodermal ridge in mouse and zebrafish embryos. Knock down of bhlha9 in zebrafish resulted in shortening of the pectoral fins.

Conclusions Genomic duplications encompassing BHLHA9 are associated with SHFLD and non-Mendelian inheritance characterised by a high degree of nonpenetrance with sex bias. Knock-down of bhlha9 in zebrafish causes severe reduction defects of the pectoral fin, indicating a role for this gene in limb development.

Distal limb reduction defect is the generic term for a larger group of congenital malformations which affect the autopod. Among these, splithand/foot malformations (SHFM) constitute a separate group. SHFM is extremely variable in its phenotypic expression between families, within families, and even between limbs of a single patient, ranging from syndactyly and oligodactyly to the most severe expression-monodactyly with only a single phalanx. Ectrodactyly is not exclusively observed in humans but has also been described in other species such as chicken, ${ }^{1}$ frogs ${ }^{2}$ and mice. ${ }^{3}$ Besides genetic factors studies in rodents show that environmental factors-for example, treatment of pregnant rodents with retinoic acid or cadmium-cause ectrodactyly. ${ }^{4}$ The teratogenic effect in these cases is due to induction of apoptosis of cells from the apical ectodermal ridge (AER). ${ }^{4}$ Currently, six different loci/mutations (SHFM1-6) have been associated with non-syndromic SHFM phenotypes in humans. These include dominant mutations in TP63 (SHFM4, MIM 605289), recessive mutations in WNT1OB (SHFM6, MIM 225300), as well as genomic duplications on 10q24 (SHFM3, MIM 246560), and deletions and translocations on 7q (SHFM1, MIM 183600)(reviewed in Duijf et al ${ }^{6}$ ). The genetic cause is still unknown in SHFM2 (MIM 313350) which maps to Xq26, and SHFM5 (MIM 606708) which maps to 2q31. However, a large number of cases remain unsolved. Due to reduced penetrance and variable expressivity, inheritance patterns are often difficult to interpret, and dominant as well as recessive and X-linked inheritance has been postulated. Besides these isolated forms of SHFM, syndromic forms of ectrodactyly have been described such as EEC syndrome (MIM 129900), which is characterised by ectrodactyly, ectodermal dysplasia, and cleft lip/ palate. Missense mutations in TP63 (MIM 603273) cause EEC syndrome. ${ }^{78}$ In some families SHFM is associated with long bone deficiency involving the tibia and fibula, in which case the condition is referred to as split-hand/foot malformation with long bone deficiency (SHFLD, MIM 119100). Unusual inheritance patterns have made this condition less accessible for mapping approaches with subsequent disease gene discovery. ${ }^{9}$

Based on our previous results indicating that copy number variations (CNVs) may cause congenital malformations, ${ }^{10-13}$ we performed high 
resolution array comparative genomic hybridisation (CGH) analysis (1M oligo array, Agilent Technologies, Santa Clara, California, USA) in patients with SHFLD. Blood sampling and extraction of DNA was performed by standard methods. All participants gave their consent for molecular testing. This study was approved by the Charité Universitätsmedizin Berlin ethics committee. In a pedigree suggestive of X-linked inheritance (family 10, figure $1 \mathrm{~A}$ and supplementary figure 2), we identified a duplication of $180 \mathrm{~kb}$ on chromosome 17p13.3 (figure 1A). We further analysed a large Brazilian family previously mapped to the same region ${ }^{14}$ and identified a $110 \mathrm{~kb}$ microduplication (family 16, figure 1A). To identify further families and to test the frequency of $17 \mathrm{p} 13$ duplications, we screened a cohort of another 54 families with non-syndromic SHFM including another 11 cases with long bone deficiency (SHFLD). Clinical manifestations and limb anomalies in these patients are summarised in table 1 . In this cohort, 17p duplications were identified in $30 \%(17 / 56)$ of the families, TP63 mutations were detected in 9\% (5/56), and 10q24 duplications in 20\% (11/56). Of the remaining 23 families of this cohort, nine individuals were screened by array CGH and no deletions at the SHFM1 locus on chromosome $7 \mathrm{q}$ were detected. Balanced rearrangements were not excluded. Note that five of 43 patients $(12 \%)$ presenting with SHFM and no tibial/long bone involvement showed $17 \mathrm{p} 13.3$ duplications, which is comparable to the frequency of TP63 mutations in our non-syndromic SHFM cohort. In summary, 17 p13.3 duplications have to be considered the most common aetiology of SHFLD.

A Chromosome 17p13.3

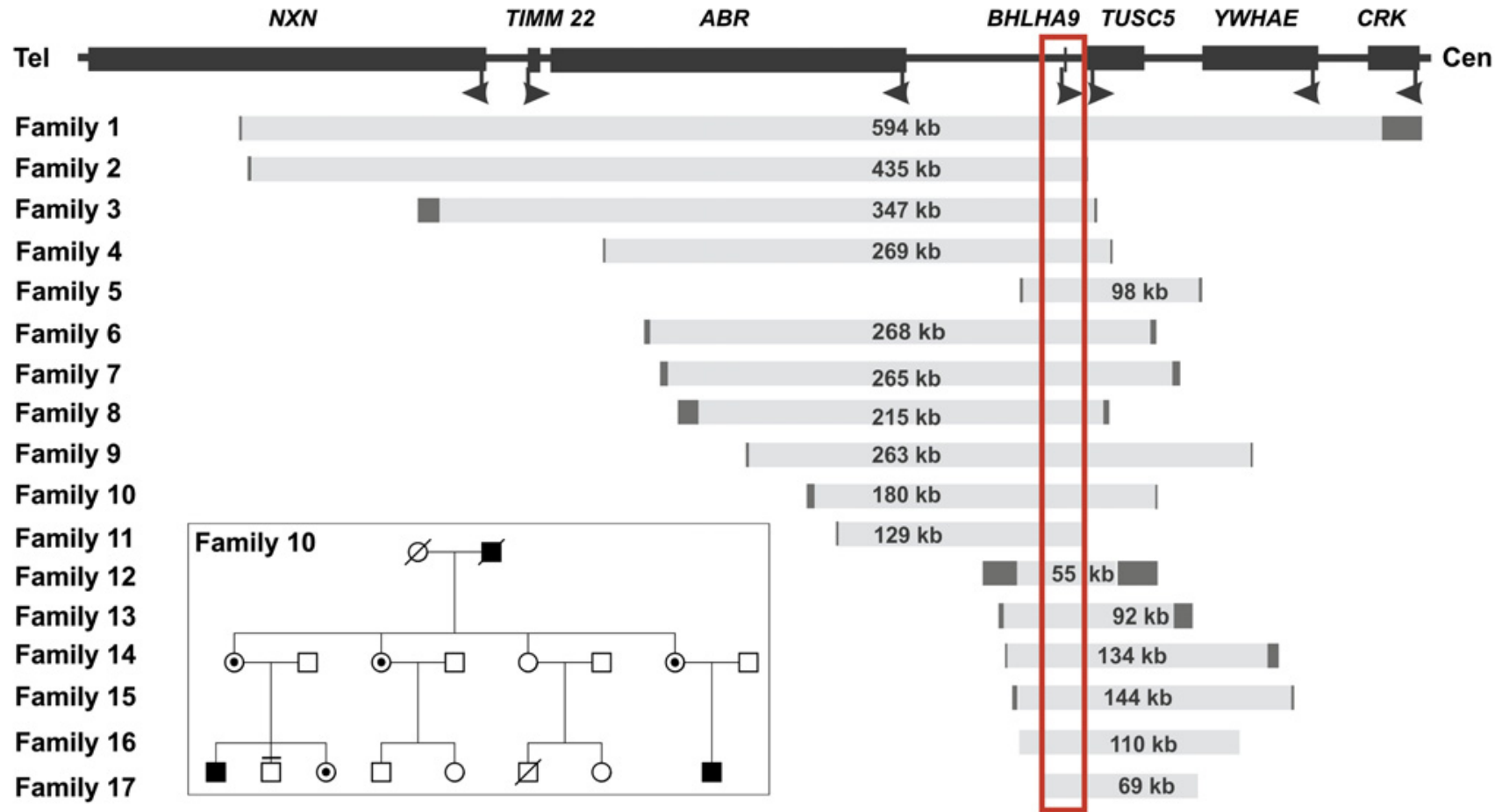

B

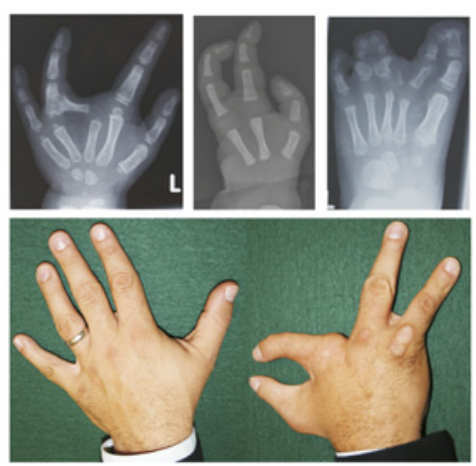

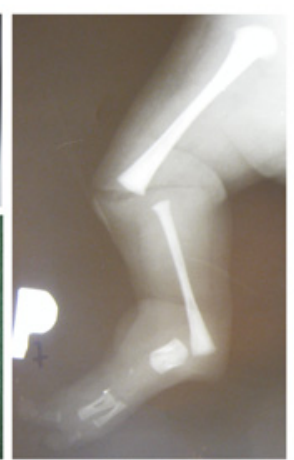

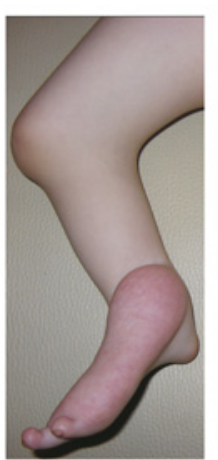

C

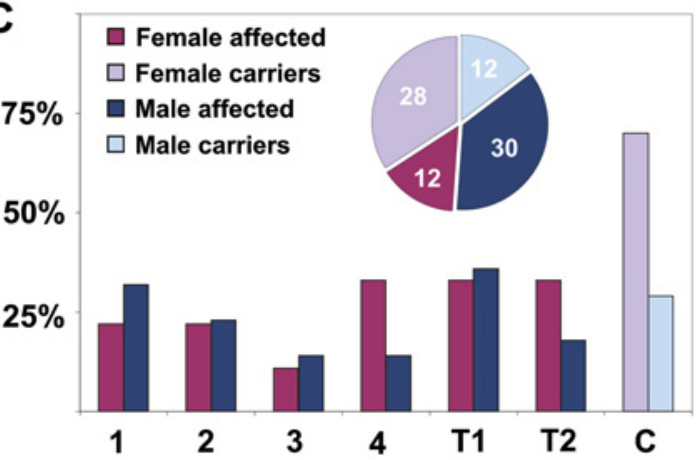

Figure 1 Schematic overview of microduplications in 17p13.3 associated with split-hand/foot malformation with long bone deficiency (SHFLD) (families 1-17), clinical phenotypes, and distribution. (A) The smallest region of overlap (SRO) is indicated by the red box and encompasses BHLHA9. Pedigree family 10 is example of a pedigree showing dominant inheritance with reduced penetrance and sex bias. Duplicated regions-light grey; breakpoint regions-dark grey. Genomic positions according to hg18. (B) Examples of clinical phenotypes. Note strong intraindividual variability, for example, split right hand and only mildly affected left hand (lower left). Typical presentation of tibial aplasia (right). (C) Pie chart showing distribution of $17 \mathrm{p}$ duplication among healthy and affected carriers (82 individuals from 17 families). Bar chart illustrates range in phenotypic variability in 31 affected from 16 families. Each bar depicts the percentage of affected for the specific phenotype with respect to patients' gender. As underlying scoring criteria we chose the number of affected limbs (that is, 1-4) and the presence of tibia hemimelia (T1, unilateral; $T 2$, bilateral). In addition, sex distribution in healthy carriers $(C)$ is shown. 
Table 1 Clinical manifestations in our split-hand/foot malformations (SHFM) cohort

\begin{tabular}{|c|c|c|c|c|c|c|c|}
\hline \multirow[b]{3}{*}{ Family } & \multirow[b]{3}{*}{ Genotype } & \multicolumn{6}{|c|}{ Phenotype of index patient } \\
\hline & & \multicolumn{2}{|c|}{ Ectrodactyly } & \multicolumn{2}{|c|}{ Monodactyly } & \multirow{2}{*}{$\begin{array}{l}\text { Tibia aplasia/ } \\
\text { hypoplasia }\end{array}$} & \multirow[b]{2}{*}{ Other features } \\
\hline & & Hands & $\overline{\text { Feet }}$ & Hands & Feet & & \\
\hline 2 & & NA & NA & NA & NA & + & NA \\
\hline 3 & & $+/+$ & $+/+$ & $-1-$ & $-1-$ & $+/+$ & - \\
\hline 4 & & $+/+$ & $-/+$ & $-1-$ & $-1-$ & $-1-$ & - \\
\hline 7 & & $+/+$ & $+/+$ & $-1-$ & $-1-$ & $+/+$ & - \\
\hline 8 & & $+/+$ & $-/+$ & $-1-$ & $-1-$ & $+/+$ & - \\
\hline 9 & & $+/+$ & $-1-$ & $-1-$ & $-1-$ & $-1-$ & - \\
\hline 10 & & $+/+$ & $+/+$ & $-1-$ & $-1-$ & $-1-$ & - \\
\hline 11 & & $+/-$ & $-1-$ & $-1-$ & $-1-$ & $+/-$ & - \\
\hline 12 & & $+/+$ & $-/+$ & $-1-$ & $-1-$ & $+/+$ & - \\
\hline 17 & & $+/+$ & $+/+$ & $-1-$ & $-1-$ & $-1-$ & - \\
\hline 18 & 10q24 duplication & $+/+$ & $+/+$ & $-1-$ & $-1-$ & $-1-$ & Oligodontia, learning disability \\
\hline 19 & & $+/+$ & $+/+$ & $-1-$ & $-1-$ & $-1-$ & - \\
\hline 20 & & + & + & $-1-$ & $-1-$ & $-1-$ & - \\
\hline 21 & & $-1+$ & $+/+$ & $-1-$ & $-1-$ & $-1-$ & - \\
\hline 22 & & $-1-$ & $-1-$ & $+/+$ & $+/+$ & $-1-$ & - \\
\hline 23 & & $+/+$ & $+/+$ & $-1-$ & $-1-$ & $-1-$ & Preaxial polydactyly of hand unilateral \\
\hline 24 & & $+/+$ & $+/+$ & $-1-$ & $-1-$ & $-1-$ & - \\
\hline 25 & & $+/+$ & $+/+$ & $-1-$ & $-1-$ & $-1-$ & Preaxial polydactyly of hand unilateral \\
\hline 26 & & $-/+$ & $-1-$ & $+/-$ & $+/+$ & $-1-$ & - \\
\hline 27 & & $+/+$ & $+/+$ & $-1-$ & $-1-$ & $-1-$ & Intellectual disability \\
\hline 28 & TP63 mutation & $-1-$ & $+/+$ & $-1-$ & $-1-$ & $-1-$ & - \\
\hline 37 & & $-1-$ & $+/+$ & $-1-$ & $-1-$ & $-1-$ & Aplasia/hypoplasia of fibulae \\
\hline 38 & & $-1-$ & $+/+$ & $-1-$ & $-1-$ & $-1-$ & - \\
\hline 39 & & $-1-$ & $+/+$ & $-1-$ & $-1-$ & $-1-$ & - \\
\hline 40 & & $-1+$ & $+/+$ & $-1-$ & $-1-$ & $-1-$ & - \\
\hline 41 & & $-1-$ & $+/+$ & $-1-$ & $-1-$ & $-1-$ & - \\
\hline 42 & & $-1-$ & $+/+$ & $-1-$ & $-1-$ & $-/+$ & $\begin{array}{l}\text { Hypoplasia of the third toes, hypoplasia of } \\
\text { the tibia/fibula unilateral }\end{array}$ \\
\hline 43 & & $+/+$ & $+/+$ & $-1-$ & $-1-$ & $-1-$ & Reduction defects of lower legs and forearms \\
\hline 44 & & $+/+$ & $+/+$ & $-1-$ & $-1-$ & $-1-$ & - \\
\hline 45 & & $+/+$ & $+/+$ & $-1-$ & $-1-$ & $-1-$ & - \\
\hline 46 & & $+/+$ & $+/+$ & $-1-$ & $-1-$ & $-1-$ & Renal duplication, bifid uvula \\
\hline 47 & & $+/+$ & $+/+$ & $-1-$ & $-1-$ & $-1-$ & Hypoplasia of fibulae \\
\hline 48 & & $+/+$ & $+/+$ & $-1-$ & $-1-$ & $-1-$ & Aplasia of fibulae \\
\hline 49 & & $-/+$ & $-1-$ & $+/-$ & $-1-$ & $-1-$ & Hypoplasia of ulna unilateral \\
\hline 50 & & + & + & $-1-$ & $-1-$ & $-1-$ & Short stature, ectodermal dysplasia \\
\hline 51 & & $+/+$ & $-1-$ & $-1-$ & $-1-$ & $-1-$ & $\begin{array}{l}\text { Postaxial polydactyly of hands, sparse blond hair, } \\
\text { small teeth, cleft palate, dysplastic kidneys, imperforate anus, } \\
\text { micropenis, microcephaly, intellectual disability }\end{array}$ \\
\hline 52 & & $-1-$ & $+/-$ & $-1-$ & $-1-$ & $-1-$ & - \\
\hline 53 & & + & + & $-1-$ & $-1-$ & $-1-$ & Familial cleft lip/palate \\
\hline 54 & & $+/+$ & $+/+$ & $-1-$ & $-1-$ & $-1-$ & Short stature, mild intellectual disability \\
\hline 55 & & + & + & $-1-$ & $-1-$ & $-1-$ & Light hair and skin \\
\hline 56 & & $+/+$ & $-1-$ & $-1-$ & $-1-$ & $-1-$ & Pre-axial polydactyly of feet \\
\hline
\end{tabular}

\footnotetext{
+ , present; - , absent; $\pm / \pm$, right/left.
} 
The duplication breakpoints in the 17 families were nonrecurrent and duplication sizes varied from $69 \mathrm{~kb}$ to $594 \mathrm{~kb}$ (figure 1A; data submitted to DECIPHER database). The overlapping region was identified between positions 1117153 and 1128992 corresponding to $\sim 11.8 \mathrm{~kb}$ of sequence (red box, figure 1A). The duplications were arranged in direct tandem orientation as demonstrated by breakpoint sequencing (supplementary figure 1). In the 17 families all of the 42 affected individuals showed the duplication and 40 individuals were identified as non-affected carriers by quantitative real-time PCR (pedigrees in supplementary figure 2).

Duplications overlapping with the smallest region of overlap (SRO) are not listed in the database of genomic variants. In the literature, however, 17p13.3 duplications have been described in patients with mental retardation and autistic manifestations. ${ }^{15} 16$ The duplications in some of the described individuals overlap the critical region described here, but skeletal defects were not observed. The absence of limb anomalies in these individuals is probably due to non-penetrance. Our cases with duplications on $17 p$ have been assessed for developmental delay and autistic features by pediatricians and/or clinical geneticists. Since none of our affected individuals presented with autistic features or developmental delay, it is likely that different risk alleles are located within the distal region of chromosome $17 p$

Recently, Armour et al ${ }^{17}$ described three SHFLD families with overlapping duplications on chromosome 17p13.3. Taken together with our results, the SRO is located between 1117153-1128916 and encompasses only a single putative gene, BHLHA9, with a one-exon open reading frame which translates to a protein containing the characteristic structural motif of basic helix loop helix (bHLH) transcription factors. Transcription factors of the bHLH type are involved in regulation of cell proliferation, differentiation, and various developmental processes (reviewed by Jones ${ }^{18}$ ). To investigate the natural expression pattern of BHLHA9, we identified the mouse and zebrafish orthologues and performed whole mount in situ hybridisation at different embryonic stages according to standard procedures (mouse transcript BCO48728; zebrafish transcript wu:fb99e06). These experiments revealed that Bhlha9 expression in both species is restricted to the distal limb bud mesenchyme underlying the AER, supporting the idea of a role in limb development (figure 2A-I). We did not observe any other major expression domains. To investigate the role of BHLHA9 in limb development we performed morpholino (MO) knockdown experiments in zebrafish embryos ( $A B$ stock maintained at $28.5^{\circ} \mathrm{C}$ ). Antisense custom-morpholino oligonucleotides (MO_bhlha9) were targeted around the start codon of bhlha9 (5'-CAGACGCTCGCTGGAGTCATGGATA-3') and ordered from Gene Tools (Gene Tools, LLC, Philomath, Oregon, USA). For negative control experiments the standard control oligonucleotide (MO_scrambled) from Gene Tools was used. Embryos were injected with $5 \mu \mathrm{M}$ per embryo of $1 \mathrm{mM}$ solution at the one cell stage ( $M O$ _bhlha9: $n=83 ; \mathrm{MO}_{-}$scrambled: $\mathrm{n}=42$ ). Uninjected embryos $\overline{(n}=92)$ from the same pool were used as wild-type controls (WT). Embryos were fixed at different time points with $4 \%$ paraformaldehyde overnight at $4^{\circ} \mathrm{C}$, subsequently washed twice with PBST, and stored in $100 \%$ methanol. All $83 \mathrm{MO}$ _bhlha9 injected embryos showed severely truncated pectoral fins compared to controls at $72 \mathrm{~h}$ post-fertilisation (hpf) (figure 2J-O), indicating that Bhlha9 is important for limb development in a dosage dependent manner.

Outgrowth of the limb is regulated via diverse mechanisms including signals from the AER, an ectodermal cell lining, and the underlying progress zone (PZ). Without the AER the limb does not grow because cells of the $\mathrm{PZ}$ do not receive the necessary signal for proliferation as shown by experimental removal of the AER in chicken embryos. ${ }^{19}$ The importance of the AER in limb outgrowth and its relation to SHFM has been demonstrated by investigations of the mouse mutant Dactylaplasia (Dac), which shows an ectrodactyly phenotype due to degeneration of the central AER starting after developmental stage E11.5. ${ }^{20}$ As a consequence, a reduction in proliferation of the underlying mesenchyme was observed which ultimately results in missing central rays and a phenotype resembling SHFM. Thus, SHFM is proposed to result from a failure to maintain AER activity in the median region of the autopod. Based on the phenotypic similarities between cases with $17 \mathrm{p} 13.3$ duplication, Dac and other SHFM mouse models (reviewed in Duijf et $a l^{6}$ ), we hypothesise that the transcription factor BHLHA9 regulates target genes important for survival and/or proliferation of cells in the $\mathrm{PZ}$. This regulation might either be under control of the AER or BHLHA9 may influence the AER directly. Since we observed pectoral fin outgrowth in the morpholino, we conclude that the initial induction of the AER is not influenced by the knockdown of bhlha9. However, the massive truncation of the pectoral fins suggests that further growth of the fin is severely disturbed, possibly due to interference with AER maintenance. In this scenario a change in dosage of BHLHA9 induced by the morpholino in zebrafish or by the duplication in patients may disturb the signalling network and the interactions between AER and PZ in the limb bud.

We observed high phenotypic variability in 31 cases with 17 p13.3 duplication in which clinical data were available. A scoring system that quantifies the degree of pathology in each limb was applied (figure 1B,C). The results show that nine of 31 affected $(29 \%)$ have only one affected limb (score=1), in six $(19 \%)$ all limbs are affected (score=4), and in 11 (35\%) unilateral (T1) or seven (23\%) bilateral tibial hemimelia (T2) was present in addition to SHFM (figure 1C). Interestingly more than half of the women with tibial involvement have both lower limbs affected (T2; figure 1C). This observation suggests that females, when affected, are generally more severely affected. The common clinical feature in all affected individuals was ectrodactyly of one or more extremities. While tibial involvement was present in approximately $60 \%$ of affected $17 p$ duplication carriers, it has so far not been described in any patient with 10q24 duplication or TP63 mutations. Thus, tibia hemimelia is a strong indicator of $17 p$ duplication as the underlying genetic cause.

We studied affected and non-affected SHFLD family members by quantitative real-time PCR and identified a high degree of non-penetrance. In total, duplications were observed in 82 individuals, of whom 42 were affected, but also in 40 unaffected carriers. Furthermore, a clear sex bias was observed resulting in more males (30/42) being affected than females (12/42) (pie chart figure $1 \mathrm{C}$ ). Together, these effects result in a non-Mendelian inheritance pattern with reduced recurrence risk and many mothers who are healthy carriers. Based on our calculations, the recurrence risk of unaffected carriers to have clinically affected children is $36 \%$ for male and $15 \%$ for female offspring.

The reason for this distortion is unknown. An imprinting effect is unlikely since affected individuals were observed in both paternal and maternal inheritance of the duplication. The presence of intraindividual variability suggests that stochastic factors play an important role in the pathogenesis of this condition. However, as yet unidentified modifier(s) either at the same locus or elsewhere in the genome are likely to be important as well. The sex ratio distortion could be due to a common 
Figure 2 Bhlha9 is required for limb development. (A-D) Limb specific Bhlha9 expression (mouse transcript $\mathrm{BCO48728)}$ in the distal mesenchyme below the apical ectodermal ridge (AER) is detected in forelimb and hindlimb. As a marker of the AER we used Fgf8 (E, F, I). Longitudinal sections of forelimb at stage E11.5 show Bh/ha9 expression restricted to the subridge mesenchymal layer as well as in the dorsal and ventral regions (H). The AER indicated by strong Fgf8 expression (I, arrow) shows no detectable Bhlha9 expression. WT zebrafish embryos at $60 \mathrm{~h}$ post-fertilisation (hpf) show a similar bhlha9 expression pattern in the mesenchyme of the fin field (arrow in $\mathrm{G}$; zebrafish transcript wu:fb99e06). Antisense morpholino (MO) oligonucleotides ( $\mathrm{M} 0$ bhlha9) were designed to analyse $\bar{b} h / h a 9$ function in zebrafish. At $72 \mathrm{hpf}$ overall growth was retarded in bh/ha9 M0. Pectoral fins are clearly visible in wild-type (WT) larvae ( $\mathrm{J}-\mathrm{L}$, uninjected controls) whereas the pectoral fins in bhlha9 MO injected larvae were severely truncated $(\mathrm{M}-0$, arrows).

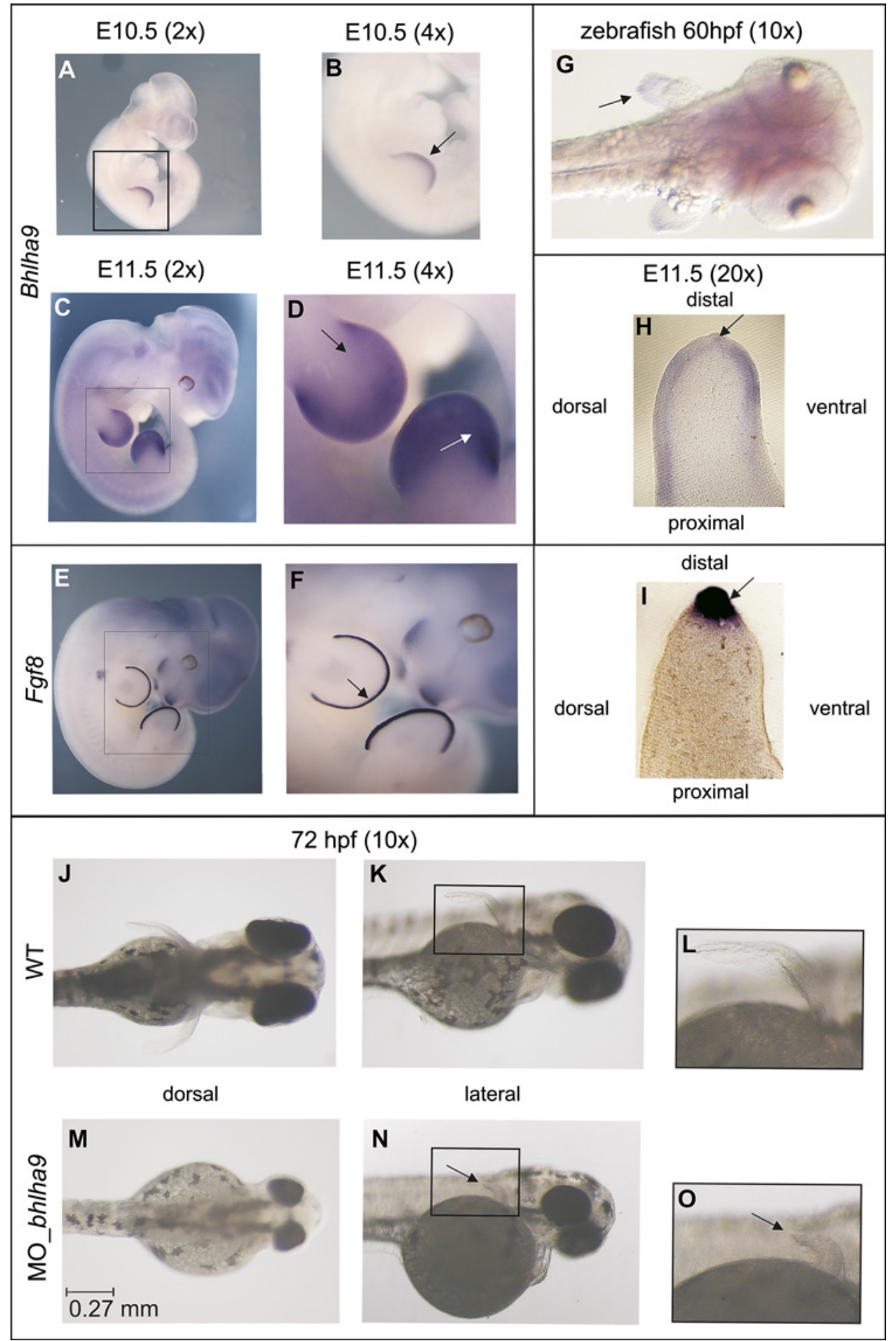

polymorphism in a hormone responsive promoter/enhancer that is 'dosage sensitive' and leads to either gene repression or expression in a sex dependent manner. Alternatively, the presence of a modifier on the $\mathrm{X}$ chromosome could lead to a 'dilution' effect in females via random $X$ inactivation. In this case, females will only be affected if both $\mathrm{X}$ chromosomes carry the modifier, or if the $\mathrm{X}$ chromosome that does not carry the modifier is over proportionally inactivated in the affected limb. Thus, the duplication on $17 \mathrm{p}$ can be seen as a susceptibility factor for SHFLD which is necessary but not sufficient for development of this malformation. A similar situation has been described for thrombocytopenia-absent-radius (TAR) syndrome
(MIM 274000). ${ }^{21}$ In TAR syndrome a microdeletion of variable size on chromosome 1q encompassing at a minimum $200 \mathrm{~kb}$ and 11 genes serves as a susceptibility factor and, similar to SHFLD, only $\sim 50 \%$ of individuals who carry the deletion show malformations. However, TAR syndrome does not show the sex bias observed in association with the $17 p$ duplication. Thus, SHFLD and TAR syndrome are examples of congenital malformations with complex, non-Mendelian inheritance. The penetrance in these conditions appears to be dependent on yet to be identified modifiers. Congenital malformations are relatively common, affecting 3-5\% of newborns. In the great majority of cases the origin is unknown. Since most of these cases are 


\section{Web resources}

- Online Mendelian Inheritance in Man (OMIM), http://www. ncbi.nlm.nih.gov/0mim/

- Database of Genomic Variants (DGV), http://projects.tcag.ca/ variation/

- Ensembl Genome Browser, http://www.ensembl.org/index. $\mathrm{html}$

- Decipher, https://decipher.sanger.ac.uk/ (Patient numbers: family 1, BER258552; family 2, BER258553; family 3, BER258555; family 4, BER258557; family 5, BER258558; family 6, BER258559; family 7, BER258560; family 8, BER258561; family 9, BER258562; family 10, BER258563; family 11, BER258564; family 12, BER258565; family 13, BER258566; family 14, BER258567; family 15, BER258569; family 16, BER258570; family 17, BER258571).

thought to be sporadic, environmental factors and/or polygenic inheritance have been considered as the underlying pathology. Our finding shows that rare CNVs can serve as a susceptibility factor for congenital disease, a mechanism which may explain increased recurrence risk in conditions otherwise considered to be sporadic.

\section{Author affiliations}

${ }^{1}$ Institute for Medical Genetics and Human Genetics, Charité-Universitätsmedizin Berlin, Berlin, Germany

${ }^{2}$ Max-Planck-Institute for Molecular Genetics, Berlin, Germany

${ }^{3}$ Department of Human Genetics, Radboud University Nijmegen Medical Centre, Nijmegen, The Netherlands

${ }^{4}$ Centro de Estudos do Genoma Humano, Departamento de Genética e Biologia Evolutiva, Instituto de Biociências, Universidade de São Paulo, São Paulo, Brazi ${ }^{5}$ Laboratório de Otorrinolaringologia/LIM32, Hospital das Clínicas, Faculdade de Medicina, Universidade de São Paulo, São Paulo, Brazil

${ }^{6}$ Department of Medical Genetics, University of Medical Sciences, Poznan, Poland ${ }^{7}$ NZOZ Center for Medical Genetics GENESIS, Poznan, Poland

${ }^{8}$ Department of Orthopaedics, Paediatric Orthopaedic Service, Kasturba Medical College, Manipal, Karnataka, India

${ }^{9}$ Institut für Humangenetik, Universitätsklinikum Jena, Jena, Germany

${ }^{10}$ Kath. Kinderkrankenhaus Wilhemstift, Hamburg, Germany

${ }^{11}$ Orthopaedic Research Laboratories, Department of Orthopaedic Surgery, The Howard Hughes Medical Institute, Children's Hospital, Boston, Massachusetts, USA ${ }^{12}$ Department of Medical Genetics, Leuven University Hospital, Leuven, Belgium

${ }^{13}$ Institute for Human Genetics, Universitätsmedizin Greifswald, Greifswald, Germany

${ }^{14}$ Institute of Human Genetics, Technische Universität München, Munich, Germany

${ }^{15}$ Institute of Human Genetics, Helmholtz Zentrum München, Neuherberg, Germany

${ }^{16}$ Department of Genetics, Directorate General of Health Affairs, Ministry of Health, Muscat, Sultanate of Oman

${ }^{17}$ Children's Hospital, Helsinki University Central Hospital, University of Helsinki, and Folkhälsan Institute of Genetics, Helsinki, Finland

${ }^{18}$ Center for Arab Genomic Studies (CAGS), Dubai, United Arab Emirates

${ }^{19}$ Department of Genetic Medicine and Development, University of Geneva Medical School, Geneva, Switzerland

${ }^{20}$ Division of Medical Genetics, Geneva University Hospitals, Geneva, Switzerland

Acknowledgements We acknowledge Randy Koll and Fabienne Trotier for excellent technical assistance.

Funding This work was supported by the Deutsche Forschungsgemeinschaft to EK and SM (grant number KL 2158/2-1). AJ was supported by a grant from the Polish Ministry of Science and Higher Education (grant number 495/N-NIEMCY/2009/0).

Competing interests None.

Patient consent Obtained.

Ethics approval Ethics committee of Charité Universitätsmedizin Berlin.

Contributors EK conducted the study and analysed the data, drafted the manuscript, approved the final version of the manuscript; SL performed the in situ hybridisation as well as zebrafish experiments, approved the final version of the manuscript; SCD carried out the clinical analysis of the cases and participated in drafting the manuscript, approved the final version of the manuscript; SS participated in in situ hybridisation experiments, approved the final version of the manuscript; CWO performed qPCR experiments, approved the final version of the manuscript; RSTA participated in analysis of family, approved the final version of the manuscript; KL provided patient samples and clinical data, approved the final version of the manuscript; RCMN provided patient samples and clinical data, approved the final version of the manuscript; AJ provided patient samples and clinical data, approved the final version of the manuscript; HS provided patient samples and clinical data, approved the final version of the manuscript; IK provided patient samples and clinical data, approved the final version of the manuscript; RH provided patient samples and clinical data, approved the final version of the manuscript; MW provided patient samples and clinical data, approved the final version of the manuscript; KD provided patient samples and clinical data, approved the final version of the manuscript; UK provided patient samples and clinical data, approved the final version of the manuscript; MH provided patient samples and clinical data, approved the final version of the manuscript; AR provided patient samples and clinical data, approved the final version of the manuscript; OM provided patient samples and clinical data, approved the final version of the manuscript; MN provided patient samples and clinical data, approved the final version of the manuscript; UR provided patient samples and clinical data, approved the final version of the manuscript; SEA contributed to design of the study and critically revised the manuscript, approved the final version of the manuscript; DH carried out the clinical analysis of the cases and critically revised the manuscript, approved the final version of the manuscript; SM participated in the design of the study and drafted the manuscript, approved the final version of the manuscript.

Provenance and peer review Not commissioned; externally peer reviewed.

Data sharing statement Primer sequences not given in the text are available upon request.

\section{REFERENCES}

1. Abbott UK, MacCabe JA. Ectrodactyly: a new embryonic lethal mutation in the chicken. J Hered 1966;57:207-11.

2. Ouellet M, Bonin J, Rodrigue J, DesGranges JL, Lair S. Hindlimb deformities (ectromelia, ectrodactyly) in free-living anurans from agricultural habitats. J Wildl Dis 1997;33:95-104.

3. Chai CK. Dactylaplasia in mice a two-locus model for development anomalies. $J$ Hered 1981:72:234-7.

4. Sulik KK, Dehart DB. Retinoic-acid-induced limb malformations resulting from apical ectodermal ridge cell death. Teratology 1988;37:527-37.

5. Hovland DN Jr, Cantor RM, Lee GS, Machado AF, Collins MD. Identification of a murine locus conveying susceptibility to cadmium-induced forelimb malformations. Genomics 2000;63:193-201.

6. Duijf PH, van Bokhoven H, Brunner HG. Pathogenesis of split-hand/split-foot malformation. Hum Mol Genet 2003;12(Spec No 1):R51-60.

7. van Bokhoven H, Hamel BC, Bamshad M, Sangiorgi E, Gurrieri F, Duijf PH, Vanmolkot KR, van Beusekom E, van Beersum SE, Celli J, Merkx GF, Tenconi R, Fryns JP, Verloes A, Newbury-Ecob RA, Raas-Rotschild A, Majewski F, Beemer FA, Janecke A, Chitayat D, Crisponi G, Kayserili H, Yates JR, Neri G, Brunner HG. p63 Gene mutations in eec syndrome, limb-mammary syndrome, and isolated split hand split foot malformation suggest a genotype-phenotype correlation. Am J Hum Genet 2001;69:481-92.

8. Celli J, Duijf P, Hamel BC, Bamshad M, Kramer B, Smits AP, Newbury-Ecob R, Hennekam RC, Van Buggenhout G, van Haeringen A, Woods CG, van Essen AJ, de Waal R, Vriend G, Haber DA, Yang A, McKeon F, Brunner HG, van Bokhoven H. Heterozygous germline mutations in the p53 homolog p63 are the cause of EEC syndrome. Cell 1999;99:143-53.

9. Naveed M, Nath SK, Gaines M, Al-Ali MT, Al-Khaja N, Hutchings D, Golla J, Deutsch S, Bottani A, Antonarakis SE, Ratnamala U, Radhakrishna U. Genomewide linkage scan for split-hand/foot malformation with long-bone deficiency in a large Arab family identifies two novel susceptibility loci on chromosomes 1q42.2-q43 and 6q14.1. Am J Hum Genet 2007;80:105-11.

10. Dathe K, Kjaer KW, Brehm A, Meinecke P, Nürnberg P, Neto JC, Brunoni D, Tommerup N, Ott CE, Klopocki E, Seemann P, Mundlos S. Duplications involving a conserved regulatory element downstream of BMP2 are associated with brachydactyly type A2. Am J Hum Genet 2009;84:483-92.

11. Kantaputra PN, Klopocki E, Hennig BP, Praphanphoj V, Le Caignec C, Isidor B, Kwee ML, Shears DJ, Mundlos S. Mesomelic dysplasia Kantaputra type is associated with duplications of the HOXD locus on chromosome 2q. Eur J Hum Genet 2010;18:1310-14.

12. Klopocki E, Ott CE, Benatar N, Ullmann R, Mundlos S, Lehmann K. A microduplication of the long range SHH limb regulator (ZRS) is associated with triphalangeal thumb-polysyndactyly syndrome. J Med Genet 2008;45:370-5.

13. Klopocki E, Lohan S, Brancati F, Koll R, Brehm A, Seemann P, Dathe K, Stricker S, Hecht J, Bosse K, Betz RC, Garaci FG, Dallapiccola B, Jain M, Muenke M, Ng VC, Chan W, Chan D, Mundlos S. Copy-number variations involving the IHH locus are associated with syndactyly and craniosynostosis. Am J Hum Genet 2011;88:70-5. 
14. Lezirovitz K, Maestrelli SR, Cotrim NH, Otto PA, Pearson PL, Mingroni-Netto RC. A novel locus for split-hand/foot malformation associated with tibial hemimelia (SHFLD syndrome) maps to chromosome region 17p13.1-17p13.3. Hum Genet 2008:123:625-31

15. Bruno DL, Anderlid BM, Lindstrand A, van Ravenswaaii-Arts C, Ganesamoorthy D, Lundin J, Martin CL, Douglas J, Nowak C, Adam MP, Kooy RF, Van der Aa N, Reyniers E, Vandeweyer G, Stolte-Dijkstra I, Dijkhuizen T, Yeung A, Delatycki M, Borgström B, Thelin L, Cardoso C, van Bon B, Pfundt R, de Vries BB, Wallin A, Amor DJ, James PA, Slater HR, Schoumans J. Further molecular and clinical delineation of co-locating 17p13.3 microdeletions and microduplications that show distinctive phenotypes. J Med Genet 2010;47:299-311.

16. Bi W, Sapir T, Shchelochkov OA, Zhang F, Withers MA, Hunter JV, Levy T, Shinder V, Peiffer DA, Gunderson KL, Nezarati MM, Shotts VA, Amato SS, Savage SK, Harris DJ, Day-Salvatore DL, Horner M, Lu XY, Sahoo T, Yanagawa Y, Beaudet AL, Cheung SW, Martinez S, Lupski JR, Reiner 0. Increased LIS1 expression affects human and mouse brain development. Nat Genet 2009;41:168-77.
17. Armour CM, Bulman DE, Jarinova O, Rogers RC, Clarkson KB, Dupont BR, Dwivedi A, Bartel FO, McDonell L, Schwartz CE, Boycott KM, Everman DB, Graham GE. 17p13.3 microduplications are associated with split-hand/foot malformation and long-bone deficiency (SHFLD). Eur J Hum Genet 2011;19:1144-51.

18. Jones S. An overview of the basic helix-loop-helix proteins. Genome Biol 2004;5:226.

19. Summerbell D. A quantitative analysis of the effect of excision of the AER from the chick limb-bud. J Embryol Exp Morphol 1974;32:651-60.

20. Crackower MA, Motoyama J, Tsui LC. Defect in the maintenance of the apical ectodermal ridge in the Dactylaplasia mouse. Dev Biol 1998;201:78-89.

21. Klopocki E, Schulze H, Strauss G, Ott CE, Hall J, Trotier F, Fleischhauer S, Greenhalgh L, Newbury-Ecob RA, Neumann LM, Habenicht R, König R, Seemanova E, Megarbane A, Ropers HH, Ullmann R, Horn D, Mundlos S. Complex inheritance pattern resembling autosomal recessive inheritance involving a microdeletion in thrombocytopenia-absent radius syndrome. Am J Hum Genet 2007;80:232-40.

\section{Have confidence in your decision making.}
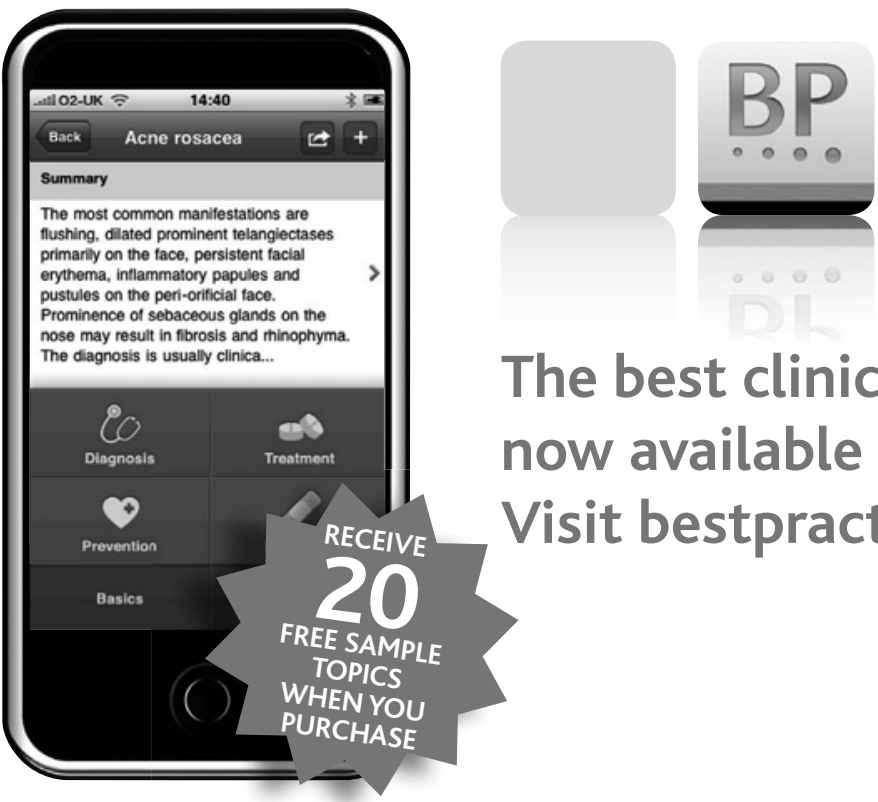

The best clinical decision support tool is now available as an app for your iPhone. Visit bestpractice.bmj.com/app

\section{clinicians $\bullet$ medical students $\bullet$ nurses $\bullet$ healthcare practitioners}




\section{Duplications of BHLHA9 are associated with ectrodactyly and tibia hemimelia inherited in non-Mendelian fashion}

Eva Klopocki, Silke Lohan, Sandra C Doelken, et al.

$J$ Med Genet 2012 49: 119-125 originally published online December 6, 2011

doi: 10.1136/jmedgenet-2011-100409

Updated information and services can be found at:

http://jmg.bmj.com/content/49/2/119.full.html

These include:

\section{Data Supplement "Supplementary Data"}

http://jmg.bmj.com/content/suppl/2011/12/06/jmedgenet-2011-100409.DC1.html

References This article cites 21 articles, 6 of which can be accessed free at: http://jmg.bmj.com/content/49/2/119.full.html\#ref-list-1

Email alerting Receive free email alerts when new articles cite this article. Sign up in service the box at the top right corner of the online article.

Topic Articles on similar topics can be found in the following collections

Collections

Reproductive medicine (470 articles)

Immunology (including allergy) (507 articles)

Notes

To request permissions go to:

http://group.bmj.com/group/rights-licensing/permissions

To order reprints go to:

http://journals.bmj.com/cgi/reprintform

To subscribe to BMJ go to:

http://group.bmj.com/subscribe/ 